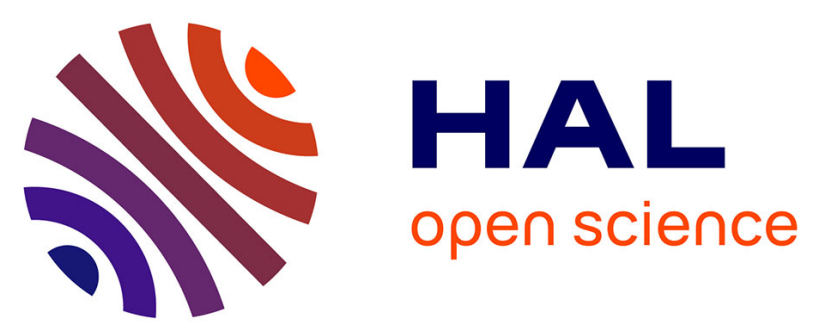

\title{
Kinematic Analysis of Magnetic Continuum Robots Using Continuation Method and Bifurcation Analysis
}

Quentin Peyron, Quentin Boehler, Kanty Rabenorosoa, Bradley J. Nelson, Pierre Renaud, Nicolas Andreff

\section{- To cite this version:}

Quentin Peyron, Quentin Boehler, Kanty Rabenorosoa, Bradley J. Nelson, Pierre Renaud, et al.. Kinematic Analysis of Magnetic Continuum Robots Using Continuation Method and Bifurcation Analysis. IEEE Robotics and Automation Letters, 2018, 3 (4), pp.3646-3653. 10.1109/LRA.2018.2855803 . hal-02126172

\section{HAL Id: hal-02126172 \\ https://hal.science/hal-02126172}

Submitted on 10 May 2019

HAL is a multi-disciplinary open access archive for the deposit and dissemination of scientific research documents, whether they are published or not. The documents may come from teaching and research institutions in France or abroad, or from public or private research centers.
L'archive ouverte pluridisciplinaire HAL, est destinée au dépôt et à la diffusion de documents scientifiques de niveau recherche, publiés ou non, émanant des établissements d'enseignement et de recherche français ou étrangers, des laboratoires publics ou privés. 


\title{
Kinematic analysis of magnetic continuum robots using continuation method and bifurcation analysis
}

\author{
Q. Peyron ${ }^{1,2}$, Q. Boehler ${ }^{3}$, K. Rabenorosoa ${ }^{2}$, B. J. Nelson ${ }^{3}$, P. Renaud ${ }^{1}$, N. Andreff ${ }^{2}$
}

\begin{abstract}
Magnetic continuum robots (m-CR) have grown interest in several applicative contexts that take benefits from their high flexibility and remote control. When submitted to external magnetic fields, m-CR exhibit large elastic deformations, which may lead to a highly non-linear and complex behavior that is yet difficult to analyze. This paper aims to provide a tool to improve the understanding of $\mathrm{m}$-CR kinematics. To do so, a numerical approach composed of continuation methods and bifurcation analysis is proposed. The numerical tool is first described. Qualitative and quantitative results are further introduced by computing multiple equilibrium branches and bifurcations of mCR configurations within homogeneous and inhomogeneous magnetic fields. Our simulations are ultimately compared to experimental data using a magnetic navigation system.
\end{abstract}

Index Terms-Kinematics, flexible robots.

\section{INTRODUCTION}

$\mathbf{M}$ AGNETIC continuum robots (m-CR) have grown interest in minimally invasive surgeries such as catheter-based cardiac procedures [1, [2] and eye surgery [3. They are composed of flexible sections with permanent magnets [1] or micro-coils [2] embedded at their tip. Robot deflections are driven with magnetic fields, which produce magnetic torques on the magnet. This distal actuation allows m-CR to generate tip forces while having a soft body, safe for interactions with human tissues. Magnetic fields and gradients are produced with a magnetic navigation system (MNS). Various architectures have been considered for its implementation: MRI systems [2], fixed coils controlled in current such as [4, [1], and magnetic sources displaced with robot architectures such as permanent magnets [5, [6] or coils [7].

Manuscript received: February, 24th, 2018; Revised April, 23th, 2018; Accepted June, 22nd, 2018.

This paper was recommended for publication by Editor Dezhen Song upon evaluation of the Associate Editor and Reviewers' comments. This work was supported by the French National Agency for Research (NEMRO ANR-14-CE17-0013), the Investissements d'Avenir (Robotex ANR-10-EQPX-44, Labex CAMI ANR-11LABX-0004 and Labex ACTION ANR-11-LABX-0001-01), Aviesan FLI infrastructure and by the Swiss National Science Foundation through grant number 200021165564 .

${ }^{1}$ Authors are with the ICube Lab, UDS-CNRS-INSA, 300 bd Sébastien Brant - Illkirch, France. pierre.renaud@unistra.fr

${ }^{2}$ Authors are with the FEMTO-ST Institute, Univ. Bourgogne Franche Comté, CNRS, Besançon, France. quentin.peyron@femto-st.fr

${ }^{3}$ Authors are with the Multi-Scale Robotics Lab, ETH Zurich, Tannenstrasse 3, 8092 Zurich, Switzerland. qboehler@ethz.ch

Digital Object Identifier (DOI): see top of this page.

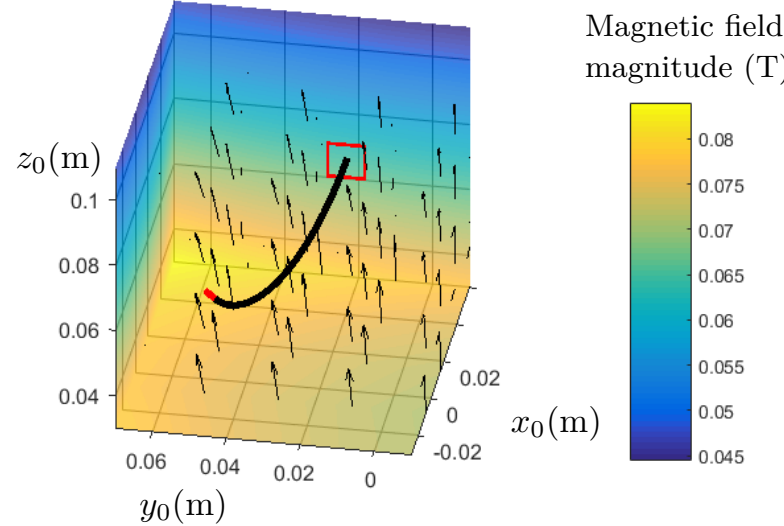

Figure 1: Representation of a one-magnet $\mathrm{m}-\mathrm{CR}$ controlled with the CardioMag MNS [1. A desired magnetic field of magnitude $0.08 \mathrm{~T}$ is generated in $[0,0,0]$. The $\mathrm{m}-\mathrm{CR}$ is clamped at the center of the red square.

The use of $\mathrm{m}$-CR is hampered by the existence of unstable configurations which can be reached during manipulation, as observed and studied in 8 in the case of a planar robot. The kinematic model has in addition locally multiple solutions between which the robot may snap dangerously. These phenomena are likely to appear for m-CR evolving in space, which is the general situation of main practical interest. This needs therefore to be studied in order to improve the safety while exploiting the full workspace of these robots. This is a difficult task first because m-CR are expected to adopt complex and non trivial 3D shapes due to magnetic and gravity forces. Second, the magnetic field produced by a MNS is intrinsically not homogeneous, i.e. it is not constant but varies continuously within the MNS workspace [9]. These inhomogeneities are depicted in term of magnetic field magnitude in Fig. 1 1 where a specific m-CR and MNS have been considered within the scope of this work. The impact of corresponding perturbated magnetic torques and forces is not yet assessed while the robot kinematics are directly affected by these inhomogeneities.

In this paper, kinematic analysis is considered with a numerical approach. The $\mathrm{m}-\mathrm{CR}$ is analogous to an elastic rod, with a highly non linear behavior due to spatial deformations and external loadings, and with multiple configurations for a given magnetic field. Establishing the behavior of such system is a well known problem in nonlinear elastic mechanics [10], which needs to be solved 


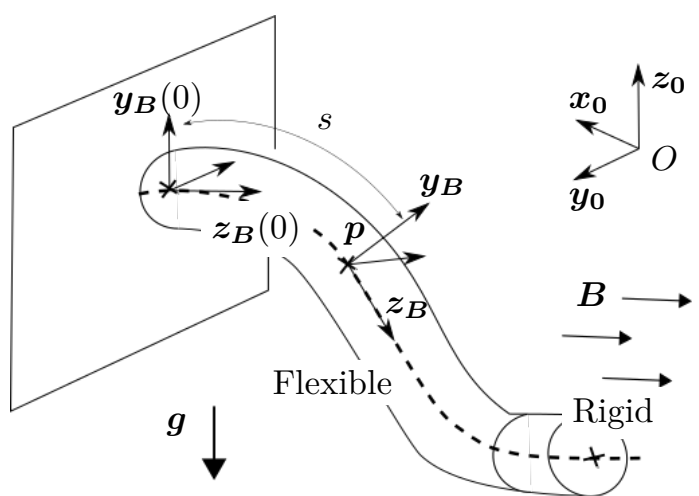

Figure 2: Spatial representation of the m-CR, with reference frame $\mathcal{R}_{0}$, local frame $\mathcal{R}_{B}$, and external magnetic field $\boldsymbol{B}$.

numerically in a general case. Continuation methods are of particular interest to establish equilibrium configurations of rods [11, 12 by determining connected sets of equilibria called branches [13. A prediction and correction process is being used, so that complex solutions of a model can be obtained without a priori knowledge on them. The existence of multiple solutions appears as bifurcations between branches of solutions, which are commonly studied to obtain information about the local behavior of rods [10]. Coupling of continuation method and bifurcation analysis exists and was proven to be efficient for studying snapping phenomena for concentric tube robots [14] and buckling of planar rods subject to magnetic forces [15]. A combination of these methods therefore appears of great interest for the analysis of $\mathrm{m}-\mathrm{CR}$.

We propose in this paper to use continuation methods and bifurcation analysis to give better understanding of spatial m-CR kinematics considering the magnetic field inhomogeneities commonly created by MNS in practice. The interest of this proposition is demonstrated in four steps, as follows. A kinematic model of $\mathrm{m}-\mathrm{CR}$ is first derived in section II and casted in a discrete form suitable for numerical analysis with the selected numerical framework. The use of continuation method and bifurcation analysis is then described in section III The ability of the proposed approach to assess m-CR behavior is demonstrated qualitatively in a third step, in section [V] on a mCR subject to homogeneous magnetic field. Additionally, new insights about the m-CR kinematics in two complex case studies are provided. Quantitative assessment of the prediction capacity in the presence of inhomogeneous fields is finally performed in the same situations in section $\mathrm{V}$ by comparison with experimental data, before concluding.

\section{KINEMATICS}

The kinematic model aims to describe the evolution of the m-CR configuration at the equilibrium according to the magnetic field $\boldsymbol{B}$ used to control the robot. Several kinematic models have been proposed in prior works to describe the behavior of loaded continuum robots and elastic rods. They are usually derived following two main approaches: the balance between internal and external forces and torques, on the one hand [16, and the first variation of the total potential energy, on the other hand [12]. In this paper, we use a kinematic model obtained essentially by following the different steps presented in [12]. Additional and relevant assumptions specific to m-CR as well as a different representation of m-CR configurations are considered.

\section{A. System description and assumptions}

Without loss of generality, we consider here a m-CR composed of a flexible section of length $L_{f}$ at which tip a rigid permanent magnet is fixed as previously introduced in [8], 15], 11, 2. The magnet constitutes a rigid section of length $L_{r}$. The robot depicted in Fig. 2 exhibits a bended shape in space due to the action of gravity and the magnetic torques and forces acting on the magnet. In addition to the assumption considered in [12, we assume that no torsion occurs. This assumption is valid when considering axial magnets as it is the case here. The m-CR tip is then free to rotate about its axis, releasing torsional stresses. We consider the magnetic torques and forces to be induced by the magnetic field $\boldsymbol{B}$ evaluated at the magnet center. Variations of the magnetic field within the magnet are neglected.

\section{B. $m$-CR configuration}

The m-CR configuration is described by the robot backbone position, orientation and curvature, which vary along the backbone that is parametrized with the arc length $s$. Dependency on $s$ is not mentioned in the following for sake of compactness. The backbone position is denoted $\boldsymbol{p}$ and is expressed in the MNS reference frame denoted $\mathcal{R}_{0}\left(O, \boldsymbol{x}_{\mathbf{0}}, \boldsymbol{y}_{\mathbf{0}}, \boldsymbol{z}_{\mathbf{0}}\right)$. The robot orientation is parameterized with a Bishop frame $\mathcal{R}_{B}\left(\boldsymbol{p}, \boldsymbol{x}_{\boldsymbol{B}}, \boldsymbol{y}_{\boldsymbol{B}}, \boldsymbol{z}_{\boldsymbol{B}}\right)$ where $\boldsymbol{z}_{\boldsymbol{B}}$ is tangent to the backbone. The rotation between $\mathcal{R}_{0}$ and $\mathcal{R}_{B}$ is represented with Euler angles arranged in a ZYX sequence [17], and gathered in a vector $\boldsymbol{a}$ so that $\boldsymbol{a}=$ $\left[\begin{array}{lll}a_{X} & a_{Y} & a_{Z}\end{array}\right]^{T}$. This representation provides a minimal state representation of the orientation and the resulting geometrical singularities are not encountered in the analysis performed throughout this work. The backbone curvature is represented with a Darboux vector expressed in $\mathcal{R}_{B}$ and is denoted $\boldsymbol{u}_{\boldsymbol{B}}$. From construction of $\mathcal{R}_{B}$, the coordinate along $\boldsymbol{z}_{\boldsymbol{B}}$ of $\boldsymbol{u}_{\boldsymbol{B}}$ equals always 0 , so that $\boldsymbol{u}_{\boldsymbol{B}}=\left[\begin{array}{lll}u_{B x} & u_{B y} & 0\end{array}\right]$.

Position, orientation and curvature vectors constitute a redundant representation of the robot configuration. They are bound with the following geometric relations [17]:

$$
\begin{gathered}
\boldsymbol{a}^{\prime}=\boldsymbol{M}^{\boldsymbol{T}} \boldsymbol{u}_{\boldsymbol{B}} \quad \text { with } \boldsymbol{M}=\left[\begin{array}{ccc}
1 & 0 & -s_{Y} \\
0 & c_{X} & s_{X} c_{Y} \\
0 & -s_{X} & c_{X} c_{Y}
\end{array}\right] \\
\boldsymbol{p}^{\prime}={ }^{\boldsymbol{B}} \boldsymbol{R}_{\mathbf{0}}\left[\begin{array}{lll}
0 & 0 & 1
\end{array}\right]^{T}
\end{gathered}
$$

where prime exponent denotes the derivation with respect to $s, c_{X}$ and $c_{Y}\left(s_{X}\right.$ and $\left.s_{Y}\right)$ are respectively the cosine 
(sine) of $a_{X}$ and $a_{Y}$, and ${ }^{\boldsymbol{B}} \boldsymbol{R}_{\mathbf{0}}$ is the rotation matrix transforming $\mathcal{R}_{B}$ in $\mathcal{R}_{0}$.

\section{Mechanical equilibrium}

The actuation input $\boldsymbol{B}$ relates to the m-CR configuration by equilibrium equations. These express the balance between the magnetic forces $f_{m}$ and torques $\tau_{m}$ produced by $\boldsymbol{B}$, the gravity forces $f_{g}$ and the internal wrenches due to elastic deformation. They respectively depend on the magnetic dipole moment $m_{B}$, the mass $M$ and the stiffness matrix $K_{B}$ expressed in $\mathcal{R}_{B}$, with $k_{b}$ and $k_{t}$ the bending and torsional stiffnesses. These parameters are assumed to differ between the flexible and rigid sections, and are thus considered as piecewise constant functions of $s$. External forces can therefore be seen as piecewise constant distributed forces which are expressed in $\mathcal{R}_{0}$ :

$$
\left\{\begin{aligned}
\boldsymbol{f}_{\boldsymbol{g}} & =L^{-1} M \boldsymbol{g} \\
\boldsymbol{\tau}_{\boldsymbol{m}} & =L^{-1}\left(\left({ }^{\boldsymbol{B}} \boldsymbol{R}_{\mathbf{0}} \boldsymbol{m}_{\boldsymbol{B}}\right) \times \boldsymbol{B}\right) \\
\boldsymbol{f}_{\boldsymbol{m}} & =L^{-1} \nabla\left(\left({ }^{\boldsymbol{B}} \boldsymbol{R}_{\mathbf{0}} \boldsymbol{m}_{\boldsymbol{B}}\right) \cdot \boldsymbol{B}\right)
\end{aligned}\right.
$$

where $\boldsymbol{g}$ is the gravitational constant and $L=L_{f}, \forall s \in$ $\left[0, L_{f}\right]$ and $L=L_{r}$ otherwise.

Following [12, the equilibrium equations are derived through the formulation of the potential energy of the whole robot, which first variation with respect to m-CR configuration variables $\boldsymbol{p}, \boldsymbol{a}$ and $\boldsymbol{u}_{\boldsymbol{B}}$ is then computed. Accounting for the two geometric constraints (1) and (2), two multiplier vectors denoted $\boldsymbol{\lambda}_{\boldsymbol{a}}$ and $\boldsymbol{\lambda}_{p}$ respectively are introduced. They represent the internal wrenches mentioned before, $\boldsymbol{\lambda}_{\boldsymbol{p}}$ being homogeneous to internal forces expressed in $\mathcal{R}_{0}$ and $\boldsymbol{\lambda}_{\boldsymbol{a}}$ to internal moments expressed in $\mathcal{R}_{B}$. The equilibrium equations are then written:

$$
\left\{\begin{array}{l}
\boldsymbol{K}_{B} \boldsymbol{u}_{B}=\boldsymbol{\lambda}_{a} \\
\boldsymbol{M}^{T} \boldsymbol{\lambda}_{a}^{\prime}=-\boldsymbol{M}^{T}\left({ }^{B} \boldsymbol{R}_{0}^{T}\right) \frac{\tau_{m}}{L}-S \boldsymbol{\lambda}_{\boldsymbol{a}}-\boldsymbol{T} \boldsymbol{\lambda}_{p} \\
\boldsymbol{\lambda}_{\boldsymbol{p}}^{\prime}=-\frac{\left(\boldsymbol{f}_{\boldsymbol{m}}+\boldsymbol{f}_{\boldsymbol{g}}\right)}{L}
\end{array}\right.
$$

where

$$
\boldsymbol{S}=\frac{d \boldsymbol{M}^{T}}{d s}-\left[\frac{\partial \boldsymbol{M} \boldsymbol{a}^{\prime}}{\partial \boldsymbol{a}}\right]^{T} \text { and } \boldsymbol{T}=\left[\frac{\partial^{\boldsymbol{B}} \boldsymbol{R}_{\mathbf{0}}\left[\begin{array}{ll}
0 & 0
\end{array}\right]^{T}}{\partial \boldsymbol{a}}\right]^{T}
$$

The m-CR proximal end is clamped at a position $\boldsymbol{p}_{\mathbf{0}}$ and with an orientation $\boldsymbol{a}_{\mathbf{0}}$. Considering no other external forces applied at the robot tip, the previous differential equations are constrained by the following boundary conditions:

$$
\left\{\begin{array}{l}
\boldsymbol{a}(0)=\boldsymbol{a}_{\mathbf{0}} \\
\boldsymbol{p}(0)=\boldsymbol{p}_{\mathbf{0}} \\
\boldsymbol{\lambda}_{\boldsymbol{a}}\left(L_{f}+L_{r}\right)=\boldsymbol{\lambda}_{\boldsymbol{p}}\left(L_{f}+L_{r}\right)=\mathbf{0}
\end{array}\right.
$$

The kinematic model of the m-CR forms a two-point boundary value problem, composed of equations (1)-(4) and of boundary conditions (6). Solutions of this model are described by a vector composed of 14 state variables which are $\boldsymbol{a}, \boldsymbol{p}, u_{B x}, u_{B y}, \boldsymbol{\lambda}_{\boldsymbol{a}}$ and $\boldsymbol{\lambda}_{\boldsymbol{p}}$.

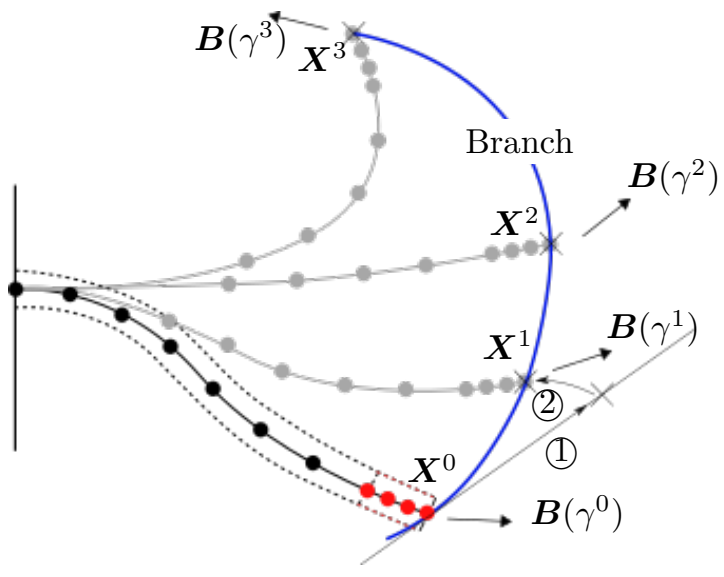

Figure 3: $\mathrm{m}-\mathrm{CR}$ configurations for in plane variation of the magnetic field. The corresponding branch of solutions obtained using prediction (1) and correction (2) steps is represented in blue. The $\mathrm{m}-\mathrm{CR}$ is represented after discretization (here, $n_{f}=7, n_{r}=4$ ).

\section{NUMERICAL METHOD}

\section{A. Discretization of $m$-CR kinematic model}

Application of the continuation method and bifurcation analysis to $\mathrm{m}-\mathrm{CR}$ requires a reformulation of the kinematic model. Following the work described in 12 for elastic rods, the robot is first discretized along its backbone. As depicted on Fig. 3, the flexible and rigid sections are represented with finite number of points denoted $n_{f}$ and $n_{r}$ respectively, indexed from the proximal to the distal ends. The state vector introduced in the previous section is evaluated at each point $k$. The derivatives of Eq. (1), 2) and (4) with respect to $s$ are then expressed with forward and backward finite differences, which gives the following geometrical constraints and equilibrium equations at point $k$ :

$$
\begin{gathered}
\left\{\begin{array}{l}
\frac{\boldsymbol{a}_{\boldsymbol{k}}-\boldsymbol{a}_{\boldsymbol{k}-\mathbf{1}}}{h_{k-1}}-\boldsymbol{M}_{\boldsymbol{k}}^{T} \boldsymbol{u}_{\boldsymbol{B}, \boldsymbol{k}}=0 \\
\frac{\boldsymbol{p}_{\boldsymbol{k}}-\boldsymbol{p}_{\boldsymbol{k}-\mathbf{1}}}{h_{k-1}}-{ }^{\boldsymbol{B}} \boldsymbol{R}_{\mathbf{0}}\left[\begin{array}{lll}
0 & 0 & 1
\end{array}\right]^{T}=0
\end{array}\right. \\
\left\{\begin{array}{l}
\boldsymbol{K}_{k} \boldsymbol{u}_{\boldsymbol{B}, \boldsymbol{k}}-\boldsymbol{\lambda}_{\boldsymbol{a}, \boldsymbol{k}}=\mathbf{0} \\
-\boldsymbol{M}_{\boldsymbol{k}}^{T} \frac{\boldsymbol{\lambda}_{\boldsymbol{a}, \boldsymbol{k}+\mathbf{1}}-\boldsymbol{\lambda}_{\boldsymbol{a}, \boldsymbol{k}}}{h_{k}}-\boldsymbol{M}_{\boldsymbol{k}}^{T}\left({ }^{\boldsymbol{B}} \boldsymbol{R}_{\mathbf{0}}^{T}\right) \\
\frac{\boldsymbol{\tau}_{\boldsymbol{m}, \boldsymbol{k}}}{L}-\boldsymbol{S}_{\boldsymbol{k}} \boldsymbol{\lambda}_{\boldsymbol{a}, \boldsymbol{k}} \\
-\frac{\boldsymbol{T}_{\boldsymbol{k}} \boldsymbol{\lambda}_{\boldsymbol{p}, \boldsymbol{k}}=\mathbf{0}}{\boldsymbol{\lambda}_{\boldsymbol{p}+\mathbf{1}}-\boldsymbol{\lambda}_{\boldsymbol{p}, \boldsymbol{k}}}-\frac{\left(\boldsymbol{f}_{\boldsymbol{m}}+\boldsymbol{f}_{\boldsymbol{g}}\right)}{L}=\mathbf{0}
\end{array}\right.
\end{gathered}
$$

where $h_{k}$ is the distance in arc length between point $k+1$ and point $k$.

The kinematic model of discrete $\mathrm{m}$-CR consists in $14\left(n_{f}+n_{r}\right)$ non-linear equations which must be solved for a given actuation field $\boldsymbol{B}$. During the manipulation of a $\mathrm{m}$-CR, $\boldsymbol{B}$ is assumed to follow a 1-dimensional path. Its components are then functions of the variable parameterizing this path, denoted $\gamma$, so that $\boldsymbol{B}=$ 
$\left[B_{x}(\gamma) \quad B_{y}(\gamma) \quad B_{z}(\gamma)\right]$. The kinematic model is thus an implicit function of the form:

$$
\boldsymbol{G}(\boldsymbol{X}, \gamma)=\mathbf{0}
$$

with $\boldsymbol{X}=\left[\begin{array}{llllll}\boldsymbol{a}_{\boldsymbol{k}} & \boldsymbol{p}_{\boldsymbol{k}} & u_{B x, k} & u_{B y, k} & \boldsymbol{\lambda}_{\boldsymbol{a}, \boldsymbol{k}} & \boldsymbol{\lambda}_{\boldsymbol{p}, \boldsymbol{k}}\end{array}\right], k=$ $1 \ldots n_{f}+n_{r}$ the vector that concatenates all discrete values of the state vector, denoted in the following as the robot configuration for sake of simplicity.

\section{B. Analysis with continuation and bifurcation}

The numerical approach used in this work is based on the computation of branches of m-CR configurations according to 1-D variations of the magnetic field. These branches correspond to a set of solutions of Eq. (9) obtained for given variations of $\gamma$. Continuation methods allow for their computation starting from an initial configuration of the robot, and applying successive variations of $\gamma$ as illustrated on Fig 3

1) Determination of $m-C R$ initial configuration: Finding an initial configuration for the m-CR, denoted $\boldsymbol{X}^{0}$ on Fig. 3, is not a trivial problem to solve considering the magnetic wrenches and the gravity forces applied on the robot. We therefore propose as an initial step to use dynamic relaxation to compute the initial configuration, as it was implemented for elastic structures subject to complex loadings in [18. The robot is first set in an arbitrary shape. Induced internal constraints are then relaxed during a virtual time evolution. The robot configuration depends thus on a virtual time, with dynamics described by the following set of second order differential equations:

$$
\boldsymbol{J} \ddot{\boldsymbol{X}}+\boldsymbol{D} \dot{\boldsymbol{X}}+\boldsymbol{G}(\boldsymbol{X}, \gamma)=\mathbf{0}
$$

where dots denotes derivatives with respect to virtual time, $\boldsymbol{J}$ and $\boldsymbol{D}$ are diagonal matrices of fictitious inertia and damping coefficients chosen here for each equation of (9) to ensure convergence. Initial configuration of the robot is further obtained by integrating Eq. (10) with an ODE solver such as ode45 as implemented in Matlab (The Mathworks, USA) until the steady state is reached. In the case the kinematic model has multiple solutions, $\boldsymbol{X}^{0}$ depends on the initial arbitrary shape chosen for dynamic relaxation.

2) Continuation and bifurcation analysis: As a second step, continuation is performed with bifurcation analysis by considering the system (10) using Matcont 19. MatCont is based on the work presented in [13] and implements the Predictor Corrector Method (PCM) for the computation of branches. Principle of the PCM is illustrated on Fig. 3 Starting from the initial point of the branch $\left(\gamma^{0}, \boldsymbol{X}^{0}\right)$, next point is predicted by stepping along the branch tangent with a given continuation step size (1). This tangent depends on the Jacobian matrices of Eq. 10 with respect to $\boldsymbol{X}$ and $\gamma$, which are evaluated numerically with finite differences. The predicted solution is then corrected using a Newton-Raphson algorithm (2) until the point $\left(\gamma^{1}, \boldsymbol{X}^{1}\right)$ is found. Numerical stability of the
Table I: Properties of the m-CR and simulation conditions.

\begin{tabular}{|c|c|c|}
\hline Section & Flexible & Rigid \\
\hline$L(\mathrm{~m})$ & 0.084 & 0.004 \\
$M(\mathrm{~kg})$ & $6.923 .10^{-4}$ & $2.110 .10^{-4}$ \\
$k_{b}\left(\mathrm{~N} . \mathrm{m}^{2}\right)$ & $1.818 .10^{-5}$ & 0.487 \\
$\boldsymbol{m}_{\boldsymbol{B}}(\mathrm{N} . \mathrm{m} / \mathrm{T})$ & {$\left[\begin{array}{lll}0 & 0 & 0\end{array}\right]$} & {$\left[\begin{array}{lll}0 & 0 & 0.263 .10^{-1}\end{array}\right]$} \\
\hline $\boldsymbol{a}_{\mathbf{0}}(\mathrm{rad})$ & \multicolumn{3}{|c|}{$\left.\begin{array}{lll}0 & -\pi / 2 & 0\end{array}\right]$} \\
$\boldsymbol{p}_{\mathbf{0}}(\mathrm{m})$ & {$\left[\begin{array}{lll}0.551 .10^{-1} & 0.226 .10^{-1} & 0.684 .10^{-1}\end{array}\right]$} \\
\hline
\end{tabular}

correction is ensured by a step size control algorithm [13, which adapts the prediction step size when needed.

A major interest of Matcont is the integration of specific functions for the detection and the localization of different type of bifurcations, including bifurcations which indicate appearance of multiple configurations such as the so-called limit points or branch points (BP) defined in [13. Matcont is also able to automatically find the direction of a new branch and follow it from a bifurcation indicating the intersection of two branches, such as a BP [13].

Association of branches and detected bifurcation forms a diagram usually denoted as a bifurcation diagram. Using the m-CR discretized model previously described and Matcont software, the m-CR behavior can be assessed through the elaboration of bifurcation diagrams. The efficiency of the method is investigated in the next two sections.

\section{PREDICTION OF M-CR BEHAVIOR}

\section{A. Simulation scenarios and parameters}

The idealized situation where the $\mathrm{m}-\mathrm{CR}$ is driven by homogeneous magnetic fields is first considered to demonstrate the interest of the method. Two case studies where critical configurations with multiple solutions to the kinematic model may exist are selected in the scope of this work. Such a situation has been identified in 8 where a planar $\mathrm{m}$-CR is driven with a magnetic field rotating around an axis orthogonal to the robot. We consider therefore two case studies where the magnetic field is rotated around the two axes orthogonal to robot proximal orientation. The field magnitude is fixed at a constant value of $0.08 \mathrm{~T}$ which corresponds to a realistic value in applications such as cardiac ablations [2]. The evolution of the magnetic field is parameterized by the variable $\gamma$ in Eq. (9) that represents the in-plane field orientation.

The considered $\mathrm{m}-\mathrm{CR}$ is composed of a $4 \mathrm{~mm}$ diameter silicone tube with a cylindrical permanent magnet placed at the tip, both components being previously used in [1. Calibrated parameters of the $\mathrm{m}-\mathrm{CR}$ are gathered in Table [1 The mass and the length of the flexible section are measured with a precision scale and a caliper respectively. The bending modulus of the flexible section is identified experimentally by fitting the kinematic model to the robot shape. This latter is measured using three visual markers equally spaced on the $\mathrm{m}-\mathrm{CR}$ and the stereo tracking system described in section V-A. Accounting for the resolution of the tracking system of $1.10^{-3} \mathrm{~m}$, the accuracy of the identification is less than $1.10^{-8} \mathrm{~N} . \mathrm{m}^{2}$. The parameters of the rigid section are based on the values 
provided by the magnet manufacturer. The discretization of m-CR geometry is performed using $\left[n_{f}, n_{r}\right]=[20,10]$. The initial configuration is then determined using dynamic relaxation with diagonal coefficients of $\boldsymbol{J}$ and $\boldsymbol{D}$ respectively equal to 125 and 300 . These parameters are determined by a trial-and-error process, that showed that a higher number of nodes does not significantly impact the numerical results, and that the selection of virtual inertia and damping matrices does not have a strong influence on the convergence of dynamic relaxation.

\section{B. Case Study \#1}

We consider the robot initially aligned with $\boldsymbol{x}_{\mathbf{0}}$ (Fig. 4b. In this first case, the magnetic field is rotated around $\boldsymbol{z}_{\mathbf{0}}$. Continuation is performed according to $\gamma$ from the initial configuration corresponding to the point 2 on the figure. As a result, it is possible to plot the altitude of the m-CR tip as a function of $\gamma$ (Fig. 4a).

For values of $\gamma$ close to 0 corresponding to points 1,2 , and 3 , the tip trajectory appears to move roughly in a plane as suggested by the horizontal tangent in $\gamma=0$ in Fig. 4a. This means that the magnetic wrenches can then balance the gravity and elastic forces.

When the field orientation increases, so do the internal wrenches due to robot stiffness. As the magnitude of $\boldsymbol{B}$ is constant, it cannot at some point balance the gravity and internal forces. This situation is observed with the simulation: the m-CR stops to bend and its tip moves progressively in the gravity direction, passing through point 4 , until it reaches a maximum displacement in $\gamma= \pm \pi$ at point 5 .

\section{Case Study \#2}

The $\mathrm{m}-\mathrm{CR}$ is in the same initial configuration. The magnetic field is now rotated around $\boldsymbol{y}_{\mathbf{0}}$ as depicted in

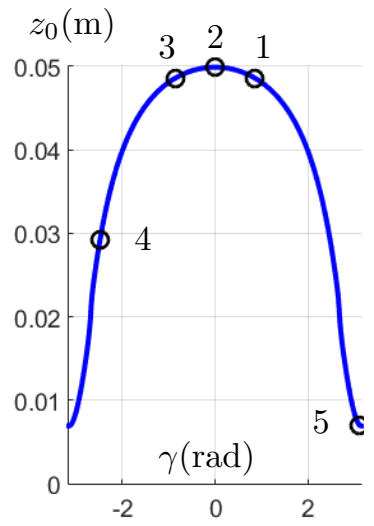

(a)

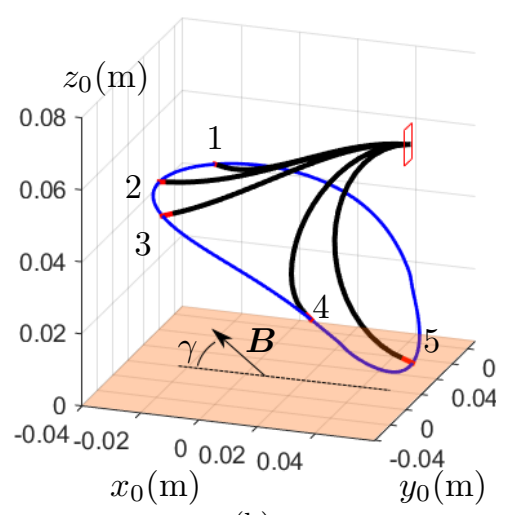

(b)
Figure 4: Results for case study \#1. The magnetic field rotates in the $\left(\boldsymbol{x}_{\mathbf{0}}, \boldsymbol{y}_{\mathbf{0}}\right)$ plane represented in orange. a) Bifurcation diagram: $\boldsymbol{z}_{\mathbf{0}}$ tip coordinate according to $\gamma . \mathrm{b}$ ) $3 \mathrm{D}$ trajectory of the robot tip.

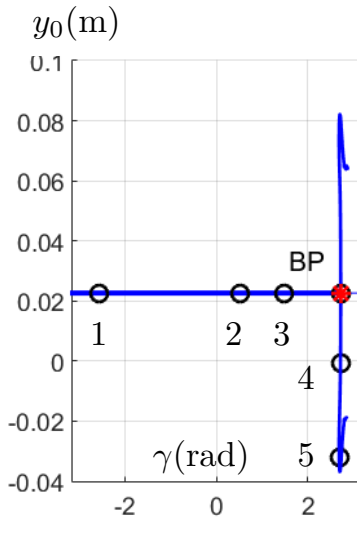

(a)

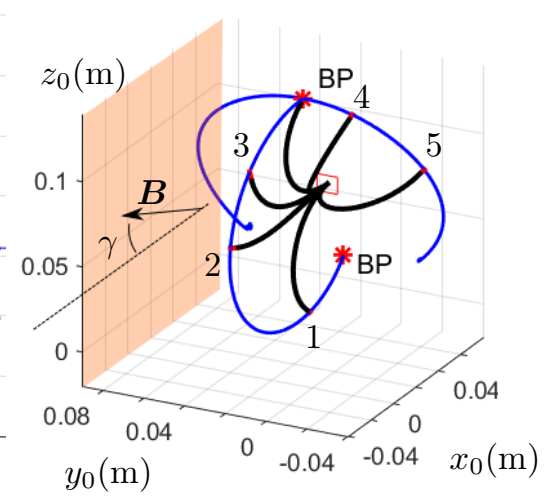

(b)
Figure 5: Results for case study \#2. The magnetic field rotates in the $\left(\boldsymbol{x}_{\mathbf{0}}, \boldsymbol{z}_{\mathbf{0}}\right)$ plane represented in orange. a) Bifurcation diagram: $\boldsymbol{y}_{\mathbf{0}}$ tip coordinate according to $\gamma . \mathbf{b}$ ) 3D trajectory of the robot tip.

Fig. 5b resulting in the bifurcation diagram illustrated in Fig. 5 a.

Starting from the initial configuration at point 2, branch of planar configurations belonging to $\left(\boldsymbol{x}_{\mathbf{0}}, \boldsymbol{z}_{\mathbf{0}}\right)$ are first computed when modulating $\gamma$, as shown with points 1 and 3. In this second case study, a BP bifurcation is encountered. The m-CR tip can follow two different paths as represented in Fig. 5a. With the proposed numerical approach, the new branch of solutions can be detected and the m-CR tip positions corresponding to this branch can be obtained. This is possible in spite of the vicinity of complex spatial configurations (points 4 and 5) which evolve with little variations of $\gamma$ between $-2.85 \mathrm{rad}$ and $-2.69 \mathrm{rad}$. The appearance of a second branch is consistent with the analysis of the external forces applied on the robot. They belong to the same plane $\left(\boldsymbol{x}_{\mathbf{0}}, \boldsymbol{z}_{\mathbf{0}}\right)$, and then do not constrain the tip to move towards a determined direction when they are balanced by the m-CR internal wrenches. In this case, out-of-plane random perturbation can then lead the m-CR to move along the second branch in one direction or the other. The robot exhibit two possible configurations for the same field orientation, which is visible at the $\mathrm{BP}$ on Fig. 5a Such analysis of m-CR behavior has never been investigated in prior works to the best of our knowledge.

The results presented in this section clearly illustrate our ability to assess 3D behavior of $\mathrm{m}-\mathrm{CR}$ in the two considered complex case studies with our numerical approach. New information about the robot kinematics are obtained, which are consistent with the m-CR physics. In addition, the combination of continuation method and bifurcation analysis allows us to detect the appearance of new branches of solutions automatically.

\section{EXPERIMENTAL COMPARISON}

The ability of our numerical tool to predict m-CR behavior subject to the magnetic field inhomogeneities induced by MNS is now assessed in two steps. The prediction 


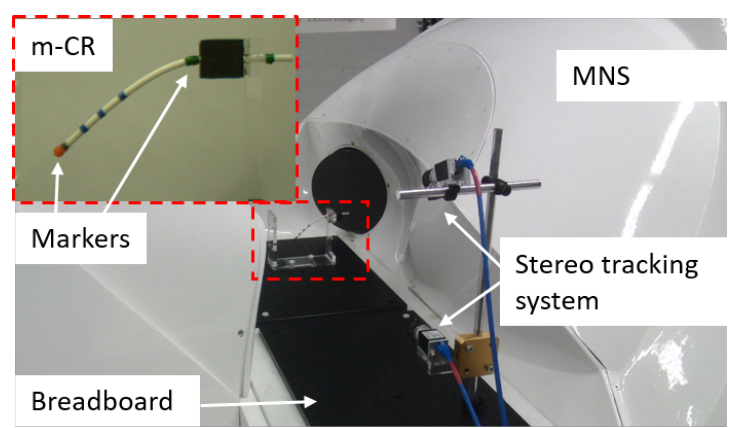

Figure 6: Experimental setup. It comprises a $4 \mathrm{~mm}$ diameter $\mathrm{m}$-CR, the MNS (CardioMag [9]) equipped with 8 fluid cooled electromagnets, and the stereo-vision tracking system.

accuracy is first evaluated by comparing numerical results with experimental data collected with the CardioMag [9], using the two case studies considered in the previous section. The tool is then used to evaluate the impact of magnetic field inhomogeneities on the $\mathrm{m}-\mathrm{CR}$, and especially in case \#2 where multiple equilibrium configurations have been identified.

\section{A. Modeling and experimental setup}

The CardioMag is composed of eight fixed coils supplied with independent controlled currents. It is thus able to generate any magnetic field or field gradient at one point of its workspace. Among the existing strategies to control $\mathrm{m}-\mathrm{CR}$ with this kind of MNS, the approach used in [20] is interesting in order to highlight impact of field inhomogeneities on the robot kinematics. A reference magnetic field $\boldsymbol{B}_{\boldsymbol{r}}$ and a null gradient are imposed at the MNS workspace center $O$ (Fig. 2 using the calibrated model presented in [9] and the actuation matrix introduced in [4] for coils current computation. This results in a different magnetic field $\boldsymbol{B}$ and in field gradient applied at the robot magnet. Errors between $\boldsymbol{B}$ and $\boldsymbol{B}_{\boldsymbol{r}}$ and gradients are therefore present all along the robot manipulation, and may have significant impact when the robot tip is far from $O$. This situation is investigated in the following.

Experiments are conducted using the setup depicted on Fig. 6. The robot proximal orientation is accurately fixed with respect to the MNS frame axis using a drilled breadboard. The proximal position is determined so that the robot tip is close to the MNS workspace center. A colored bead (6 $\mathrm{mm}$ diameter) is glued to the tip so that its position can be measured with a stereo tracking system composed of two Basler A602fc cameras $(656 \times 490$ pixels, $15 \mathrm{~Hz}$ ), with an accuracy of $1 \mathrm{~mm}$. Green markers $(5 \mathrm{~mm}$ width) are also embedded at the $\mathrm{m}-\mathrm{CR}$ proximal end to register the base position. The rotating magnetic field described in section IV] is used here as reference field $\boldsymbol{B}_{\boldsymbol{r}}$. The field orientation angle $\gamma$ is discretized in 72 steps of $5 \mathrm{~s}$ between 0 to $2 \pi \mathrm{rad}$. The step duration is chosen high enough for the robot to reach its equilibrium configuration. The MNS is then controlled through a custom $\mathrm{C}++$

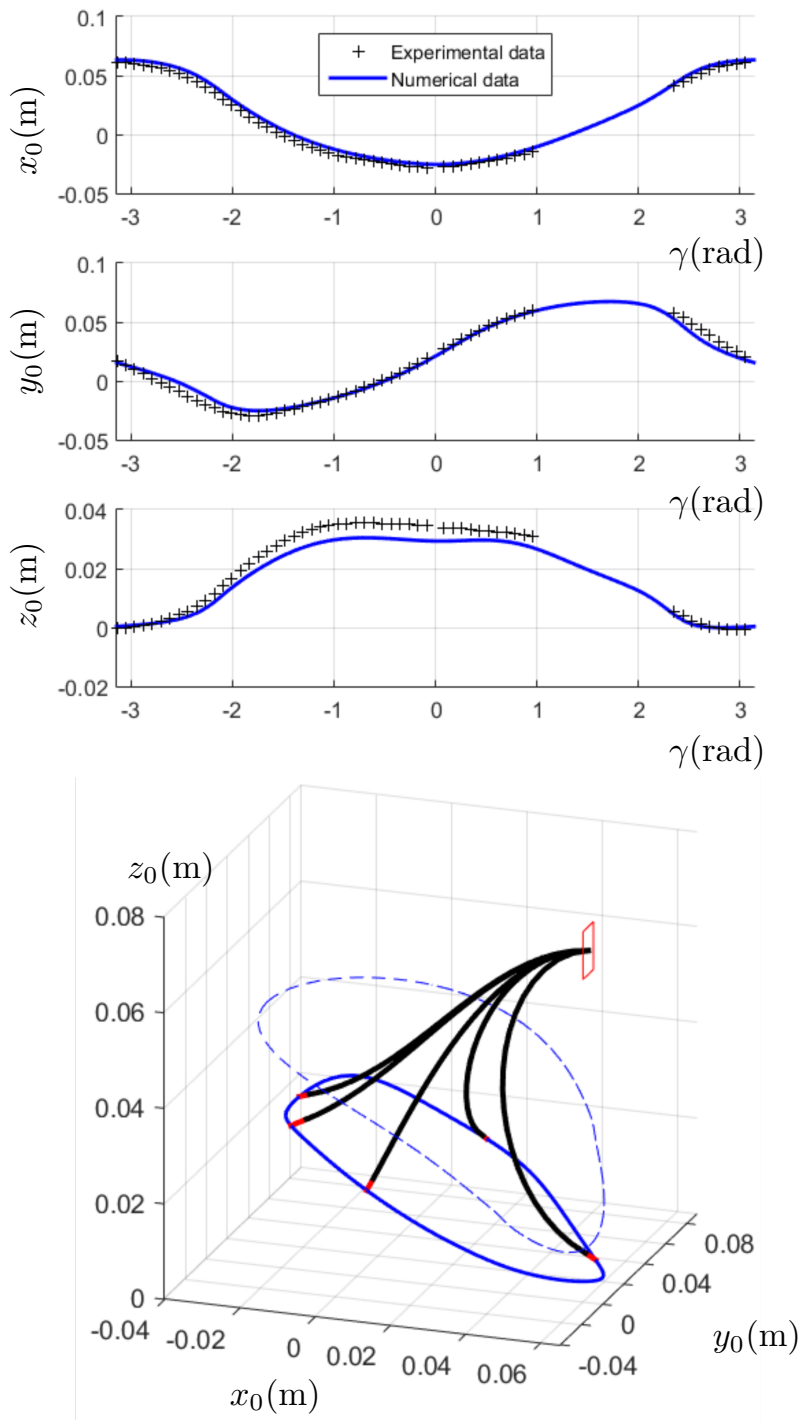

Figure 7: Experimental confrontation for $\boldsymbol{B}_{\boldsymbol{r}}$ rotating in $\left(\boldsymbol{x}_{\mathbf{0}}, \boldsymbol{y}_{\mathbf{0}}\right)$ plane. Top: Comparison between numerical and experimental results. Numerical results are in blue line and experimental tip positions are presented with black crosses. Bottom: Comparison of the robot tip trajectory computed with homogeneous (dotted line) and inhomogeneous field (plain line).

Linux program running on a $2.8 \mathrm{GHz}$ Intel Xeon W3530 processor with 12 GB RAM.

Kinematic analysis is performed using the calibrated robot parameters in Table [. We consider the MNS model parameters identified from external magnetic field measures in [9]. The bead is modeled by adding its mass at the robot tip and by extending the position of this last one by the bead radius once diagrams have been generated.

\section{B. Accuracy assessment}

The measured tip position of the $\mathrm{m}$-CR for each rotation step of $\boldsymbol{B}_{\boldsymbol{r}}$ is compared with the corresponding computed 
position. The accuracy is then computed in term of the RMS value of the difference between the two tip positions in the MNS frame $\mathcal{R}_{0}$. Errors are expressed as a percentage of the $\mathrm{m}$-CR total length which equals then $0.088 \mathrm{~m}$. Set of measured and computed positions of m-CR tip are superimposed on Fig. 7 and Fig. 8 for the two case studies. The partial absence of experimental data on Fig. 7. for $\gamma$ between 1 and $2.25 \mathrm{rad}$, is due to occlusion of the colored bead during the field rotation. The tip position error value is for each coordinate $[4.17 ; 4.61 ; 3.95] \%$ for case 1 and $[12.27 ; 6.37 ; 7.58] \%$ for case 2 . Error increase for the second case is mainly due to the S-shape region, where $\gamma \in[-2,-1.8]$ rad. Such branch shape is well known in continuum robotics to present unstable equilibrium configurations which cannot be followed experimentally [21. It induces a jump of the robot between two stable configurations, which is illustrated in our case in the accompanying video. As a consequence, the largest error occurs on the $\boldsymbol{x}_{\mathbf{0}}$ component where the S-region magnitude is the largest. Outside the S-shape region, the error values are $[6.81 ; 3.96 ; 5.93] \%$. They are then consistent with the errors obtained with existing models of loaded continuum robot using measured and manufacturer parameters, which are for instance within the same range to $4.47 \%$ of the robot length in [16. These results demonstrate the ability of the proposed numerical approach to capture the robot behavior, even for the large spatial deformation of the $\mathrm{m}-\mathrm{CR}$ considered in the two case studies and in the presence of magnetic field inhomogeneities.

\section{Impact of inhomogeneous magnetic field}

The numerical tool can be interestingly used to evaluate the impact of the field inhomogeneity on the m-CR kinematics. This can be simply performed by comparing the graphs obtained with and without considering the MNS model described in [9]. For the first case, presented in Fig. 7. the robot behavior exhibit some similarities with the one predicted before. The $\boldsymbol{z}_{\mathbf{0}}$ coordinate is almost constant for $|\gamma|<1$ and decreases dramatically until reaching a minimum value at $\gamma= \pm \pi \mathrm{rad}$. The tip trajectory is however severely distorted by field inhomogeneities, especially on $\boldsymbol{z}_{0}$ coordinate where the difference reaches $22.0 \%$ of the total robot length for $\gamma=0 \mathrm{rad}$.

As introduced in section I, these distortions are due to the m-CR tip position which does not correspond to the point $O$ where $\boldsymbol{B}_{r}$ is generated. Depending on their relative position, force and torque perturbations are created at the tip and may be critical in the bending direction of the robot in case study \#2, as explained in section IV-C. In particular, force and torque perturbations in the $\boldsymbol{y}_{\mathbf{0}}$ direction are likely to induce the expected outof-plane motion in the vicinity of the BP bifurcation. They are induced by the $\boldsymbol{y}_{\mathbf{0}}$ component of $\boldsymbol{B}$ which varies depending on the $\mathrm{m}-\mathrm{CR}$ tip position. We then propose a numerical analysis of the impact of magnetic field inhomogeneities in case study \#2 by clamping the robot at two different positions in the MNS workspace, $\boldsymbol{p}_{\mathbf{0}}$ and
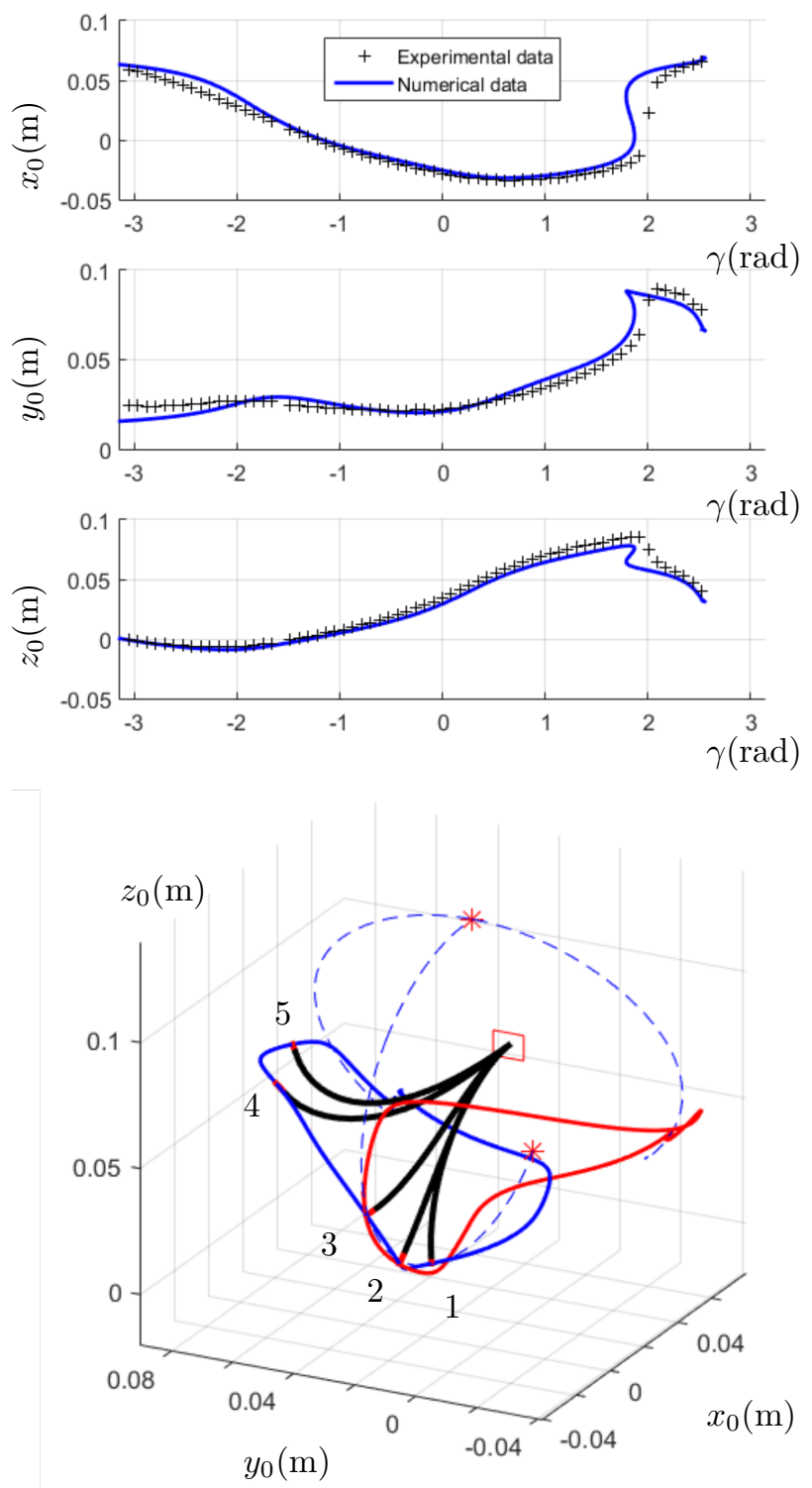

Figure 8: Experimental confrontation for $\boldsymbol{B}_{\boldsymbol{r}}$ rotating in $\left(\boldsymbol{x}_{\mathbf{0}}, \boldsymbol{z}_{\mathbf{0}}\right)$ plane. Top: Comparison between numerical and experimental results in term of tip coordinate in $\mathcal{R}_{0}$. Numerical results are represented in blue line and experimental tip position coordinates with black crosses. Bottom: Comparison of the robot tip trajectory computed with homogeneous (dotted line) and inhomogeneous field (plain line). Branches are computed for two proximal position, $\boldsymbol{p}_{\mathbf{0}}$ in blue and $\boldsymbol{p}_{\mathbf{0} \boldsymbol{b}}$ in red.

$p_{0 b}=\left[\begin{array}{lll}0.801 .10^{-1} & -0.537 .10^{-1} & 0.707 .10^{-1}\end{array}\right] \mathrm{m}$, and by relating the observed behavior with the $\boldsymbol{y}_{\mathbf{0}}$ component of $\boldsymbol{B}$.

The corresponding bifurcation diagrams generated with our numerical tool are depicted in Fig. 8, where the two clamping positions have been superimposed for the comparison. In the diagram related to $\boldsymbol{p}_{\mathbf{0}}$, the robot tip stays roughly in a plane, as shown by points 1,2 , and 3 . Then, it moves out of the plane after point 3 and seems 
to converge towards the second branch predicted with homogeneous field in the positive $\boldsymbol{y}_{\mathbf{0}}$ direction, passing through points 4 and 5 without encountering any bifurcation. The $\boldsymbol{y}_{\mathbf{0}}$ component of $\boldsymbol{B}$ is equal to $+3.25 \%$ of the magnetic field magnitude at point 2. Similarly, the robot which base is clamped at $\boldsymbol{p}_{\boldsymbol{0} \boldsymbol{b}}$ bends in the $-\boldsymbol{y}_{\mathbf{0}}$ direction when following the second branch, and the component of $\boldsymbol{B}$ is negative and equals $-4.12 \%$ for the value of $\gamma$ corresponding to point 2 . These results first highlight the fact that the $\boldsymbol{y}_{\mathbf{0}}$ component of $\boldsymbol{B}$ is decisive in the out of plane branch after bifurcation followed by the m-CR. They also show that little variations of magnetic field due to the field inhomogeneity have a high impact on the robot behavior during the field rotation.

\section{CONCLUSION}

In this paper, we used continuation methods and bifurcation analysis in order to investigate the kinematics of $\mathrm{m}$-CR. Consistent prediction of the robot behavior are obtained in two cases of spatial m-CR controlled under a homogeneous magnetic field, leading to complex deformed shape and multiple configurations. The numerical tool is also able to deal with inhomogeneous fields produced by a MNS in order to provide results as accurate as existing kinematic model of continuum robots. Our approach thus allows for an accurate and extensive analysis of $\mathrm{m}-\mathrm{CR}$ behavior in practical magnetic manipulation scenarios in the presence of magnetic field inhomogeneities.

In future work, the numerical approach presented here could be considered to investigate other scenarios of $\mathrm{m}$ CR manipulation where particular phenomena such as snapping occurs. Since the introduced kinematic model allows for arbitrary number of flexible and magnetic parts, $\mathrm{m}$-CR with two or three magnets could be considered as well. From a numerical point of view, comparison between the proposed framework and other implementations of continuation could also be performed.

\section{REFERENCES}

[1] J. Edelmann, A. J. Petruska, and B. J. Nelson, "Magnetic control of continuum devices," The International Journal of Robotics Research, vol. 36, pp. 68-85, January 2017.

[2] T. Liu and M. C. Çavuşoğlu, "Three dimensional modeling of an MRI actuated steerable catheter system," in 2014 IEEE International Conference on Robotics and Automation (ICRA), pp. 4393-4398, May 2014.

[3] F. Ullrich, S. Schuerle, R. Pieters, A. Dishy, S. Michels, and B. J. Nelson, "Automated capsulorhexis based on a hybrid magneticmechanical actuation system," in 2014 IEEE International Conference on Robotics and Automation (ICRA), pp. 4387-4392, May 2014.

[4] M. P. Kummer, J. J. Abbott, B. E. Kratochvil, R. Borer, A. Sengul, and B. J. Nelson, "OctoMag: An electromagnetic system for 5-DOF wireless micromanipulation," IEEE Transactions on Robotics, vol. 26, pp. 1006-1017, December 2010.

[5] K. R. J. Chun, E. Wissner, B. Koektuerk, M. Konstantinidou, B. Schmidt, T. Zerm, A. Metzner, R. Tilz, S. Boczor, A. Fuernkranz, F. Ouyang, and K.-H. Kuck, "Remote-controlled magnetic pulmonary vein isolation using a new irrigated-tip catheter in patients with atrial fibrillation," Circulation. Arrhythmia and Electrophysiology, vol. 3, pp. 458-464, October 2010.
[6] L. B. Kratchman, T. L. Bruns, J. J. Abbott, and R. J. Webster, "Guiding elastic rods with a robot-manipulated magnet for medical applications," IEEE Transactions on Robotics, vol. 33, pp. 227-233, February 2017.

[7] B. Véron, A. Hubert, J. Abadie, and N. Andreff, "Geometric analysis of the singularities of a magnetic manipulation system with several mobile coils," in 2013 IEEE/RSJ International Conference on Intelligent Robots and Systems, pp. 4996-5001, November 2013

[8] I. Tunay, "Modeling magnetic catheters in external fields," in The 26th Annual International Conference of the IEEE Engineering in Medicine and Biology Society, vol. 1, pp. 2006-2009, September 2004.

[9] A. J. Petruska, J. Edelmann, and B. J. Nelson, "Model-based calibration for magnetic manipulation," IEEE Transactions on Magnetics, vol. 53, pp. 1-6, July 2017.

[10] S. S. Antman, Nonlinear problems of elasticity. No. 107 in Applied mathematical sciences, New York: Springer, 2nd ed., 2005.

[11] T. J. Healey and P. G. Mehta, "Straightforward computation of spatial equilibria of geometrically exact cosserat rods," International Journal of Bifurcation and Chaos, vol. 15, pp. 949-965, Mar. 2005.

[12] A. Lazarus, J. T. Miller, and P. M. Reis, "Continuation of equilibria and stability of slender elastic rods using an asymptotic numerical method," Journal of the Mechanics and Physics of Solids, vol. 61, pp. 1712-1736, August 2013.

[13] Y. A. Kuznetsov, Elements of Applied Bifurcation Theory, vol. 112 of Applied Mathematical Sciences. Springer New York, 2004.

[14] Q. Peyron, K. Rabenorosoa, N. Andreff, and P. Renaud, "Towards more versatile concentric tube robots using stiffness modulation.," pp. 215-217, SURGETICA Conference, 2017.

[15] K. Singh, C. R. Tipton, E. Han, and T. Mullin, "Magnetoelastic buckling of an euler beam," Proc. R. Soc. A, vol. 469, p. 20130111, July 2013.

[16] D. C. Rucker, B. A. Jones, and R. J. W. III, "A Geometrically Exact Model for Externally Loaded Concentric-Tube Continuum Robots," IEEE Transactions on Robotics, vol. 26, pp. 769780, Oct. 2010.

[17] D. Cao and R. W. Tucker, "Nonlinear dynamics of elastic rods using the cosserat theory: Modelling and simulation," International Journal of Solids and Structures, vol. 45, pp. 460-477, January 2008.

[18] M. Rezaiee-Pajand and J. Alamatian, "The dynamic relaxation method using new formulation for fictitious mass and damping," Structural Engineering and Mechanics, vol. 34, no. 1, pp. 109$133,2010$.

[19] A. Dhooge, W. Govaerts, and Y. A. Kuznetsov, "MATCONT: A MATLAB Package for Numerical Bifurcation Analysis of ODEs," ACM Transactions on Mathematical Software, vol. 29, pp. 141-164, June 2003.

[20] J. Edelmann, A. J. Petruska, and B. J. Nelson, "Estimationbased control of a magnetic endoscope without device localization," Journal of Medical Robotics Research, vol. 03, p. 1850002, November 2017.

[21] P. E. Dupont, J. Lock, B. Itkowitz, and E. Butler, "Design and Control of Concentric-Tube Robots," IEEE Transactions on Robotics, vol. 26, pp. 209-225, Apr. 2010. 